terminológicamente hablando, pero de gran relevancia. Así, podemos destacar el contraste entre commence legal proceedings y sue: ambos se refieren a "demandar," aunque el primero es el término exacto y el segundo es la forma coloquial o periodística. También observamos la profunda sensibilidad de los autores hacia la evolución de los tiempos en la aparición de expresiones como "judicialización de la vida política" e "insumisión."

Sin embargo, la mayor aportación de este diccionario la constituye su carácter bilingüe real; de nuevo, una mirada a las obras existentes nos revela una segunda parte españolinglés concebida como mero apéndice, como sección de consulta rápida. En nuestro caso, la visión lingüística de los autores permite que la "segunda mitad del diccionario" sea una verdadera ayuda a la traducción inversa, tan complicada, pero sin embargo necesaria, ya que los mismos criterios de claridad y coherencia determinan una gran riqueza de ejemplos, expresiones y giros (en este sentido, la entrada de acción constituye una prueba de lo que debe hacer un diccionario para facilitar la traducción). Del mismo modo, se trata de una obra lo bastante clara y explicativa para que puede utilizarse desde el mundo anglosajón como forma de acceso al derecho español.

En resumen, se trata de una obra indispensable, no ya sólo para el traductor jurídico y todo miembro de la abogacía, sino para completar la visión del inglés empresarial de cualquier estudioso del IFE. Sin duda, la favorable acogida que ha tenido la primera edición hará que la labor de traducir ya no sea la misma después de esta obra.

Miguel Ángel Campos Pardillos

\title{
James Joyce. "Anna Livia Plurabelle" (Finnegans Wake I.VIII). Ed. bilingüe de Francisco García Tortosa. Madrid: Cátedra, 1992, 181 pp.
}

Cuando salen a la luz trabajos como el recientemente aparecido de García Tortosa sobre Finnegans Wake, uno no puede menos que alegrarse de ser universitario y académico, joyceano y lector, y de moverse además por aguas que le son harto familiares en su labor crítica. Pero muy por encima de todo ello, es cualquier amante de la lectura y de la cultura en general quien debe congratularse por la publicación de este trabajo que viene a acercar, con una ejecución que diría impecable, al cada vez menos impenetrable y desconocido James Joyce.

El trabajo que nos ocupa es en realidad dos obras bien diferenciadas y autónomas, con entidad propia, ambas de gran altura y valor académico. Sin entrar en detalles aún, éstas serían la "Introducción" y la "Traducción" propiamente dicha. El libro se divide en seis grandes apartados o capítulos, los cuatro primeros numerados y que forman parte de lo que se denomina la "Introducción": I. Joyce y Finnegans Wake; II. Finnegans Wake; III. Anna Livia; IV. La traducción de Anna Livia Plurabelle. Cada uno de ellos, a su vez, se divide en diversos apartados, ya sin titular. Les sigue la "Bibliografía" y la traducción en sí, que en edición bilingüe cierra el trabajo con el epígrafe "Anna Livia Plurabelle." Responsables de la traducción son García Tortosa, Navarrete Franco y Tejedor Cabrera, todos ellos integrantes de un activo grupo de investigación sobre Joyce de la Universidad de Sevilla. 
Quizás el trabajo encabezado por García Tortosa pudiera haberse presentado de manera ligeramente distinta: si bien el contenido queda constituido por dos bloques bien diferenciados - Bibliografía aparte-, como se decía más arriba, en la forma se podría haber marcado mejor esta distinción, agrupando, por un lado, lo que son los capítulos I, II y III, y por otro lado el que ahora es IV, "La traducción de Anna Livia Plurabelle," unido y antecediendo inmediatamente a la traducción de $F W$ I.VII como su "auténtica" y "única" introducción. Todo ello seguido, como un tercer bloque, por la Bibliografía. Pero sus razones habrán tenido García Tortosa y sus dos colaboradores para estructurar el trabajo como lo han hecho.

En lo que uno no tiene más remedio que discrepar de García Tortosa es cuando afirma, en el umbral de su sinopsis argumental de $F W$ I.vili, que no hay que olvidar "que éste no es más que el prólogo de una traducción. .." (p. 97). Por el contrario, y como se viene tratando de decir desde el principio, los tres primeros capítulos del trabajo representan y suponen una monografía en toda regla sobre Joyce y Finnegans Wake, un estudio ameno, erudito y enriquecedor incluso para los que de alguna manera puedan estar más familiarizados no sólo con la biografía sino también, y sobre todo, con el quehacer de Joyce. Es un estudio monográfico sensible a su arte; conocedor reverente, precavido, de los flirteos y engaños, siempre fieles, de Joyce con la lengua.

La estructura y organización del trabajo es igualmente loable: las diferentes partes de las que consta se ordenan, siguiendo también una técnica joyceana en Finnegans Wake, de mayor a menor, de general a particular, con disciplina casi férrea y un desarrollo lógico, de acercamiento progresivo, como sucede cuando con una cámara de cine se va pasando de planos generales a primeros planos, a veces hasta de forma imperceptible.

El capítulo I de la "Introducción" - “Joyce y Finnegans Wake" - se divide en cinco partes bien diferenciadas, en las que García Tortosa pone de manifiesto su excelente conocimiento de los materiales que maneja, fundamentalmente la biografía de Joyce y su última obra Finnegans Wake: Dublín en las obras de Joyce, su biografía y su familia, y los avatares del autor después de 1939 hasta su muerte en 1941. Esta parte, sin querer ser un tratamiento exhaustivo de la biografía de Joyce y de cómo ésta se encuentra entretejida en sus obras de una u otra forma, se convierte, por el estilo y dominio de las fuentes, en más interesante y esclarecedora que muchas introducciones al uso o estudios monográficos.

De especial relieve es la tercera sección de este capítulo, sobre todo las páginas 28 a 37. En ellas se estudia con amena erudición la presencia de Lucía Anna Joyce en Finnegans Wake a través no sólo de Issy, sino también de la madre Anna Livia Plurabelle. Se menciona y se analiza el mito universal de la Cenicienta, conectándolo con las funciones y nombres de la hija real de Joyce y de la hija en la obra. De modo que, si hemos de creer a García Tortosa, "[e]n todo lo que antecede se encuentran las claves de no pocos enigmas en Finnegans Wake y mayormente . . . la solución al laberinto por el que Joyce introdujo a su hija en la obra" (pp. 31-32).

El capítulo II titulado "Finnegans Wake" está dividido en dos apartados o secciones, la primera de la cual comienza, con muy buen criterio, con la balada de "Finnegan's," a la que sutilmente se hace ya referencia en la portada del trabajo. Como García Tortosa afirma, "Finnegans Wake debe su título y en parte el armazón estructural, que controla la 
fuerza centrífuga de la obra, a una balada popular, conocida como 'Finnegan's Wake"" (40).

En este primer apartado (pp. 40-63) el autor se sitúa, para analizarlos, en los presupuestos filosóficos de Giambattista Vico y Giordano Bruno de Nola, manteniendo que si bien el tema central de Finnegans Wake puede ser el de la circularidad de la existencia del hombre en la tierra (p. 42), el carácter cíclico ya aparece en todas y cada una de las obras anteriores de Joyce, incluido Exiliados. Pero, y esto es importante por lo más novedoso, "[l]a circularidad es una de las claves de Finnegans Wake, de eso no cabe duda, pero el final no tiene por qué llevar literalmente al principio, es más, planteado en estos términos, iría contra la idea viconiana de analogía, por la que los ciclos se repiten de modo analógico, no idéntico" (p. 47). García Tortosa termina este primer apartado haciendo una breve sinopsis argumental, que comenta y conecta con la Historia Universal como amalgama de todas las historias. Para él la esencia de Finnegans Wake radica en el "All in all" de Hamlet y Stephen, en "todo está en todo."

En la parte final del segundo capítulo, siguiendo esa progresión de la que se hablaba más arriba, e inmediatamente antes de entrar en lo que titula "Anna Livia," el autor reflexiona sobre la lengua de Finnegans Wake. Es el de la lengua en general un terreno que García Tortosa domina con facilidad, y como joyceano se siente especialmente cómodo tratándolo en esta ocasión, respaldado además por una prosa que no por reflexiva deja de ser clara y directa. Si el tabernero de Chapelizod puede representar a todos los hombres, "la lengua inglesa tendría que desempeñar la misma función: ser única y todas al mismo tiempo" (pp. 64-65).

Con el tercer capítulo se concluye, en mi opinión, lo que es una auténtica monografía de gran calidad sobre el "hecho Joyce," centrada en Finnegans Wake. Fraccionado en cinco apartados, lo más relevante se encuentra en los dos primeros, aunque hay que mencionar la sinopsis de I.VIII página por página (pp. 96-106) y una última sección, breve, referida a los posibles modelos en los que se inspiró Joyce para perfilar el personaje de Anna Livia, en especial Nora Barnacle (pp. 107-09).

En un primer momento García Tortosa trata de las diferentes versiones o textos en los que ha ido evolucionando I.viII, cómo se ha ido enriqueciendo la idea original, de modo que "el método de composición que Joyce emplea en 'Anna Livia Plurabelle,' y en Finnegans Wake, no difiere substancialmente del que emplea la abeja en la fabricación del panal" (p. 96). Reforzando el estudio realizado sobre el texto, y también la importancia de esta parte, su análisis del nombre de la protagonista - si se me permite la expresiónfemenina de la obra, y que da título al tercer capítulo del de García Tortosa, es, a pesar de su brevedad, agudo y esclarecedor incluso para los que ya se consideraban familiarizados con el tema: sucesivamente, Plurabelle (p. 90), Livia (pp. 90-93), y Anna (p. 93).

Como se ha venido diciendo de forma insistente, a partir de aquí comienza otra monografía, la traducción de $F W$ I.VIII propiamente dicha, que debía terminar, en mi opinión, con la Bibliografía cerrando todo el trabajo. El capítulo IV titulado "La traducción de 'Anna Livia Plurabelle"' es realmente una auténtica introducción que queda separada, innecesariamente por la Bibliografía general del trabajo, de aquello a lo que natrualmente debía anteceder de forma inmediata. 
Sigue siendo García Tortosa el autor de esta introducción, que en un primer momento (pp. 110-123) presenta y compara cinco traducciones de un texto representativo, cual es FW 215.31-216.05, esto es, el final de I.VIII. Las traducciones son al inglés básico de C.K. Odgen; al francés de Samuel Beckett y otros; al italiano del propio Joyce; al francés de Lavergne; al gallego de Leopoldo R. L. Rodríguez; y finalmente al castellano de García Tortosa, Navarrete Franco y Tejedor Cabrera. Y también se comenta una traducción castellana del mismo texto realizada privadamente por Silvia-Santiesteban, y una al catalán de Sobré que corresponde a $F W$ 538.18-539.08. El capítulo termina en un tono más personal (pp. 123-26) relatando como surgió la idea de la traducción y cómo ésta se llevó a cabo.

Con el mismo escrúpulo y rigor que se ha utilizado hasta el momento, la traducción de "Anna Livia Plurabelle" está cuidada, mimada hasta el detalle. El texto de $F W$ I.VIII se presenta en el original y traducido al castellano, páginas pares e impares, respectivamente, de la edición de Cátedra, cuyo contenido se corresponde exactamente con cada página y línea del original. Si bien las líneas no se numeran -siguiendo el uso de las ediciones oficiales- sí ocurre así con las páginas del original. Con respecto a la labor realizada en la traducción, sólo se puede decir que, habiendo conocido de antemano fragmentos de ella, es encomiable y respeta el proceso seguido por Joyce en su creción, lo que la hace más válida y universal.

La Bibliografía se divide en cuatro partes; fuentes primarias; general; bibliografía citada en la Introducción; y Bibliografía usada en la Traducción: nueve apretadas y densas páginas de títulos de siempre y actualizadas, que son un instrumento precioso para cualquiera, neófito o veterano, que quiera moverse por las aguas joyceanas.

"Anna Livia Plurabelle" (Finnegans Wake I.VIII), edición bilingüe de Francisco García Tortosa, es un pequeño tesoro del que todo amante de la literatura, incluso del arte en general, debe congratularse. La pulcritud de su ejecución hace que la investigación y traducción literarias adquieran nuevos y mejores valores en nuestro país, y desde luego se consolide, de una vez para siempre en el ámbito internacional, el trabajo que en el campo joyceano se viene realizando.

J. Carnero González

Francisco Javier Torres Ribelles. Eternal Shadows: Symbolism in the Theatre of W. B. Yeats. Alicante: Publicaciones de la Universidad de Alicante, 1992, 122 pp.

Eternal Shadows: Symbolism in the Theatre of W. B. Yeats is a solid study of the ideas that led to the writing of Yeats's plays and the ideas articulated in the plays themselves. It has two leading virtues: it is a timely book, and it has achieved the difficult balance sought in all scholarly writing which aims to satisfy the needs of the general reader without skimping on its duty to cogency, clarity and rigour.

As Torres explains in the early pages, one of his aims in writing Eternal Shadows was his desire to counteract the general bias which has arisen from the traditional view of W. B. Yeats as primarily a poet. Though he naturally acknowledges the high quality of 